\title{
Front Matter: Volume 7569
}

, "Front Matter: Volume 7569," Proc. SPIE 7569, Multiphoton Microscopy in the Biomedical Sciences X, 756901 (30 March 2010); doi: 10.1117/12.858684

SPIE. Event: SPIE BiOS, 2010, San Francisco, California, United States 


\title{
Multiphoton Microscopy in the Biomedical Sciences $X$
}

\author{
Ammasi Periasamy \\ Peter T. C. So \\ Karsten König \\ Editors
}

24-26 January 2010

San Francisco, California, United States

Sponsored by

SPIE

Cosponsored by

Becker \& Hickl GmbH (Germany)

Boston Electronics (United States)

Carl Zeiss Jena GmbH (Germany)

Chroma Technology (United States)

Coherent, Inc. (United States)

High Q Laser (US), Inc. (United States)

Horiba Jobin Yvon (United States)

JenLab GmbH (Germany)

Leica Microsystems (United States)

Newport-Spectra Physics (United States)

Olympus America (United States)

Omega Optical (United States)

Semrock, Inc. (United States)

Published by

SPIE

Volume 7569

Proceedings of SPIE, 1605-7422, v. 7569

SPIE is an international society advancing an interdisciplinary approach to the science and application of light. 
The papers included in this volume were part of the technical conference cited on the cover and title page. Papers were selected and subject to review by the editors and conference program committee. Some conference presentations may not be available for publication. The papers published in these proceedings reflect the work and thoughts of the authors and are published herein as submitted. The publisher is not responsible for the validity of the information or for any outcomes resulting from reliance thereon.

Please use the following format to cite material from this book:

Author(s), "Title of Paper," in Multiphoton Microscopy in the Biomedical Sciences X, edited by Ammasi Periasamy, Peter T. C. So, Karsten König, Proceedings of SPIE Vol. 7569 (SPIE, Bellingham, WA, 2010) Article CID Number.

ISSN 1605-7422

ISBN 9780819479655

Published by

SPIE

P.O. Box 10, Bellingham, Washington 98227-0010 USA

Telephone +1 3606763290 (Pacific Time) · Fax +1 3606471445

SPIE.org

Copyright (C) 2010, Society of Photo-Optical Instrumentation Engineers.

Copying of material in this book for internal or personal use, or for the internal or personal use of specific clients, beyond the fair use provisions granted by the U.S. Copyright Law is authorized by SPIE subject to payment of copying fees. The Transactional Reporting Service base fee for this volume is $\$ 18.00$ per article (or portion thereof), which should be paid directly to the Copyright Clearance Center (CCC), 222 Rosewood Drive, Danvers, MA 01923. Payment may also be made electronically through CCC Online at copyright.com. Other copying for republication, resale, advertising or promotion, or any form of systematic or multiple reproduction of any material in this book is prohibited except with permission in writing from the publisher. The CCC fee code is 1605 $7422 / 10 / \$ 18.00$.

Printed in the United States of America.

Publication of record for individual papers is online in the SPIE Digital Library.

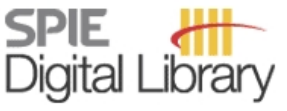

SPIEDigitallibrary.org

Paper Numbering: Proceedings of SPIE follow an e-First publication model, with papers published first online and then in print and on CD-ROM. Papers are published as they are submitted and meet publication criteria. A unique, consistent, permanent citation identifier (CID) number is assigned to each article at the time of the first publication. Utilization of CIDs allows articles to be fully citable as soon they are published online, and connects the same identifier to all online, print, and electronic versions of the publication. SPIE uses a six-digit CID article numbering system in which:

- The first four digits correspond to the SPIE volume number.

- The last two digits indicate publication order within the volume using a Base 36 numbering system employing both numerals and letters. These two-number sets start with $00,01,02,03,04$, 05, 06, 07, 08, 09, 0A, OB ... 0Z, followed by 10-1Z, 20-2Z, etc.

The CID number appears on each page of the manuscript. The complete citation is used on the first page, and an abbreviated version on subsequent pages. Numbers in the index correspond to the last two digits of the six-digit CID number. 


\section{Contents}

xi Conference Committee

KEYNOTE SESSION

756903 Plasmon-controlled fluorescence: applications to sensing and single molecule detection (Invited Paper) [7569-01]

Y. Fu, J. Zhang, J. R. Lakowicz, Univ. of Maryland School of Medicine (United States)

\section{RAMAN/CARS MICROSCOPY I}

756906 CARS and SHG microscopy of artificial bioengineered tissues (Invited Paper) [7569-05] A. Enejder, C. Brackmann, J.-O. Dahlberg, E. Vrana, P. Gatenholm, Chalmers Univ. of Technology (Sweden)

756908 High performance multimodal CARS microscopy using a single femtosecond source [7569-07]

A. F. Pegoraro, National Research Council Canada (Canada) and Queen's Univ. (Canada); A. D. Slepkov, A. Ridsdale, R. K. Lyn, D. J. Moffatt, J. P. Pezacki, National Research Council Canada (Canada); B. K. Thomas, L. Fu, L. Dong, M. E. Fermann, IMRA America, Inc. (United States); A. Stolow, National Research Council Canada (Canada) and Queen's Univ. (Canada)

756909 Frequency modulation coherent anti-Stokes Raman scattering (FM-CARS) microscopy based on spectral focusing of chirped laser pulses [7569-08]

B.-C. Chen, J. Sung, S.-H. Lim, The Univ. of Texas at Austin (United States)

\section{RAMAN/CARS MICROSCOPY II}

7569 OF Vibrational phase contrast CARS microscopy for quantitative analysis [7569-14]

M. Jurna, E. T. Garbacik, J. P. Korterik, C. Otto, J. L. Herek, H. L. Offerhaus, Univ. Twente (Netherlands)

\section{RAMAN/CARS MICROSCOPY III}

7569 ol Short-pulse fiber lasers for Raman microscopies (Invited Paper) [7569-17]

F. W. Wise, W. Renninger, S. Lefrancois, K. Kieu, M. Farrar, C. Schaffer, Cornell Univ. (United States); B. Saar, G. Holtom, S. Xie, Harvard Univ. (United States)

7569 0J A CARS solution with high temporal resolution [7569-18]

V. Lurquin, W. C. Hay, S. Landwehr, V. Krishnamachari, Leica Microsystems CMS GmbH (Germany) 
7569 OM Nonlinear Raman microscopy: improving detection through nonlinear optical interaction [7569-15]

G. I. Petrov, V. V. Yakovlev, Univ. of Wisconsin-Milwaukee (United States)

756900 Comparing coherent and spontaneous Raman scattering signals for biological imaging applications [7569-23]

B. R. Bachler, M. Cui, S. R. Nichols, J. P. Ogilvie, Univ. of Michigan (United States)

7569 OP Polarization-resolved coherent anti-Stokes Raman scattering microscopy [7569-96]

F. Munhoz, S. Brustlein, S. Brasselet, H. Rigneault, Institut Fresnel, CNRS, Aix-Marseille Univ. (France)

7569 OQ High-speed CARS spectral imaging using acousto optic tunable filter [7569-21]

M. Hashimoto, T. Minamikawa, T. Araki, Osaka Univ. (Japan)

\section{FLIM/FRET/FCS I}

7569 OS Better FLIM and FCS data by GaAsP hybrid detectors (Invited Paper) [7569-25]

W. Becker, B. Su, A. Bergmann, Becker \& Hickl GmbH (Germany)

7569 OU pH and chloride recordings in living cells using two-photon fluorescence lifetime imaging microscopy [7569-107]

M. Lahn, C. Hille, Univ. of Potsdam (Germany); F. Koberling, P. Kapusta, PicoQuant GmbH

(Germany); C. Dosche, Univ. of Potsdam (Germany)

7569 OW Regulatory assembly of the vacuolar proton pump $\mathrm{V}_{0} \mathrm{~V}_{1}$-ATPase in yeast cells by FLIM-FRET [7569-29]

S. Ernst, Univ. of Stuttgart (Germany); C. Batisse, EMBL Heidelberg (Germany); N. Zarrabi, Univ. of Stuttgart (Germany); B. Böttcher, EMBL Heidelberg (Germany) and Univ. of Edinburgh (United Kingdom); M. Börsch, Univ. of Stuttgart (Germany)

\section{FLIM/FRET/FCS II}

7569 OY Spectrally resolved fluorescence lifetime imaging: new developments and applications (Invited Paper) [7569-30]

A. Rueck, F. Dolp, ILM (Germany); B. v. Einem, C. A. F. v. Arnim, Univ. Ulm (Germany); D. Strat, ILM (Germany)

$7569 \mathrm{OZ}$ Comparison of FRET microscopy imaging techniques for studying protein-protein interactions in living cells using FRET standards [7569-27]

Y. Sun, S.-A. Seo, S. Provence, A. Periasamy, Univ. of Virginia (United States)

756913 In vivo stoichiometry monitoring of G protein coupled receptor oligomers using spectrally resolved two-photon microscopy [7569-34]

M. R. Stoneman, D. R. Singh, V. Raicu, Univ. of Wisconsin-Milwaukee (United States)

756914 New strategies to measure intracellular sodium concentrations [7569-35]

S. Dietrich, S. E. Stanca, C. G. Cranfield, B. Hoffmann, K. Benndorf, C. Biskup, Univ. Hospital Jena (Germany) 
756915 Current developments in clinical multiphoton tomography (Invited Paper) [7569-36]

K. König, JenLab GmbH (Germany) and Saarland Univ. (Germany); M. Weinigel,

H. G. Breunig, A. Gregory, P. Fischer, M. Kellner-Höfer, R. Bückle, JenLab GmbH (Germany)

756916 Long-term marker-free multiphoton imaging, targeted transfection, optical cleaning of stem cell clusters, and optical transport of microRNA with extreme ultrashort laser pulses [7569-37]

A. Uchugonova, Univ. Saarland (Germany); Z. Földes-Papp, ISS, Inc. (United States) and Medizinische Univ. Graz (Austria); G. M. Kostner, Medizinische Univ. Graz (Austria); K. König, Univ. Saarland (United States) and JenLab GmbH (Germany)

756917 Arbitrary-scan imaging for two-photon microscopy [7569-38]

E. Botcherby, C. Smith, M. Booth, R. Juškaitis, T. Wilson, Univ. of Oxford (United Kingdom)

756918 Multiphoton microscopy as a diagnostic imaging modality for lung cancer [7569-39] I. Pavlova, K. R. Hume, S. A. Yazinski, R. M. Peters, R. S. Weiss, W. W. Webb, Cornell Univ. (United States)

756919 Controllable infrared continuum source for multiphoton imaging [7569-40]

C. de Mauro, D. Alfieri, Light4Tech Firenze s.r.l. (Italy); M. Arrigoni, D. Armstrong, Coherent, Inc. (United States); F. S. Pavone, Univ. of Florence (Italy)

7569 1D Two-photon imaging with $80 \mathrm{MHz}$ and 1-GHz repetition rate Ti:sapphire oscillators [7569-43] H. Studier, H. G. Breunig, JenLab GmbH (Germany); K. König, JenLab GmbH (Germany) and Saarland Univ. (Germany)

\section{SECOND HARMONIC GENERATION I}

756911 Adaptive multiphoton and harmonic generation microscopy for developmental biology [7569-48]

A. Thayil, A. Jesacher, T. Watanabe, T. Wilson, S. Srinivas, M. J. Booth, Univ. of Oxford (United Kingdom)

7569 1K Digital holography for second harmonic microscopy [7569-50]

E. Shaffer, C. Depeursinge, École Polytechnique Fédérale de Lausanne (Switzerland)

\section{SECOND HARMONIC GENERATION II}

7569 IP Second-order susceptibility imaging with polarization-resolved second harmonic generation microscopy [7569-54]

W.-L. Chen, T.-H. Li, P.-J. Su, C.-K. Chou, P. T. Fwu, National Taiwan Univ. (Taiwan); S.-J. Lin, National Taiwan Univ. Hospital (Taiwan); D. Kim, P. T. C. So, Massachusetts Institute of Technology (United States); C.-Y. Dong, National Taiwan Univ. (Taiwan)

$75691 \mathrm{Q}$ In vivo optical virtual biopsy of human oral cavity with harmonic generation microscopy [7569-55]

M.-R. Tsai, S.-Y. Chen, National Taiwan Univ. (Taiwan); D.-B. Shieh, National Cheng-Kung Univ. (Taiwan); P.-J. Lou, National Taiwan Univ. Hospital (Taiwan); C.-K. Sun, National Taiwan Univ. (Taiwan) and Academia Sinica (Taiwan) 
7569 IR Polarization and phase pulse shaping applied to nonlinear tensorial structure read-out [7569-56]

P. Schön, Institut Fresnel, CNRS, Aix-Marseille Univ. (France); M. Behrndt, Max Planck Institute of Molecular Cell Biology and Genetics (France); D. Aït-Belkacem, H. Rigneault, S. Brasselet, Institut Fresnel, CNRS, Aix-Marseille Univ. (France)

7569 is Nonlinear optical response of the collagen triple helix and second harmonic microscopy of collagen liquid crystals [7569-57]

A. Deniset-Besseau, Lab. d'Optique et Biosciences, Ecole Polytechnique, CNRS, INSERM (France); P. De Sa Peixoto, Lab. de Chimie de la Matière Condensée, CNRS, Univ. Paris 6 (France); J. Duboisset, C. Loison, Lab. de Spectroscopie Ionique et Moléculaire, CNRS, Univ. Claude Bernard Lyon I (France); F. Hache, Lab. d'Optique et Biosciences, Ecole Polytechnique, CNRS, INSERM (France); E. Benichou, P. Brevet, Lab. de Spectroscopie Ionique et Moléculaire, CNRS, Univ. Claude Bernard Lyon I (France); G. Mosser, Lab. de Chimie de la Matière Condensée, CNRS, Univ. Paris 6 (France); M. Schanne-Klein, Lab. d'Optique et Biosciences, Ecole Polytechnique, CNRS, INSERM (France)

$75691 \mathrm{U}$ Second harmonic generation in human ovarian neoplasias [7569-59]

L. Lamonier, F. Bottcher-Luiz, L. Pietro, L. A. L. A. Andrade, A. A. de Thomaz, C. L. Machado,

C. L. Cesar, Univ. Estadual de Campinas (Brazil)

\section{TECHNOLOGY DEVELOPMENT AND APPLICATIONS II}

$75691 \mathrm{~V}$ High-throughput three-dimensional (3D) lithographic microfabrication in biomedical applications [7569-60]

D. Kim, P. T. C. So, Massachusetts Institute of Technology (United States)

7569 IW Assessment of fibrotic liver disease with multimodal nonlinear optical microscopy [7569-61] F. LU, W. Zheng, National Univ. of Singapore (Singapore); D. C. S. Tai, Institute of Bioengineering and Nanotechnology (Singapore); J. Lin, National Univ. of Singapore (Singapore); H. Yu, Institute of Bioengineering and Nanotechnology (Singapore) and National Univ. of Singapore (Singapore); Z. Huang, National Univ. of Singapore (Singapore)

$756912 \quad$ Multimodal nonlinear microscopy at $1.5 \mu \mathrm{m}$ [7569-64]

C. Zhan, C. Joo, Q. Li, GE Global Research (United States); M. Y. Berezin, W. J. Akers, Y. Ye, S. Achilefu, Washington Univ. School of Medicine (United States); S. Yazdanfar, GE Global Research (United States)

756920 Multicolor excitation two-photon microscopy: in vivo imaging of cells and tissues [7569-76] D. Li, W. Zheng, J. Y. QU, Hong Kong Univ. of Science and Technology (Hong Kong, China)

756922 The analysis of fluorophore orientation by multiphoton fluorescence microscopy [7569-66] J. M. Leeder, D. L. Andrews, Univ. of East Anglia (United Kingdom)

756923 Two-photon microscopy with dynamic focusing objective using a liquid lens [7569-67] K.-S. Lee, P. Vanderwall, J. P. Rolland, The Institute of Optics, Univ. of Rochester (United States)

756926 Pulse shaping for reducing photodamage in multiphoton microscopy [7569-125]

D. Pestov, Y. Andegeko, V. V. Lozovoy, M. Dantus, Michigan State Univ. (United States) 
756927 Real-time molecular imaging of organelles in living cell by multifocus excitation CARS microscope [7569-70]

T. Minamikawa, T. Araki, M. Hashimoto, Osaka Univ. (Japan)

756928 In situ observation of collagen thermal denaturation by second harmonic generation microscopy [7569-71]

C.-S. Liao, Z.-Y. Zhuo, J.-Y. Yu, P.-H. G. Chao, S.-W. Chu, National Taiwan Univ. (Taiwan)

756929 Spatio-temporal control in multiphoton fluorescence laser-scanning microscopy (Best Poster Award) [7569-72]

A. K. De, D. Roy, D. Goswami, Indian Institute of Technology Kanpur (India)

7569 2A Discrimination of collagen in normal and pathological dermis through polarization second harmonic generation [7569-73]

P.-J. Su, W.-L. Chen, J.-B. Hong, T.-H. Li, R.-Jr Wu, C.-K. Chou, S.-J. Lin, C.-Y. Dong, National

Taiwan Univ. (Taiwan)

7569 2B Multispectral autofluorescence lifetime imaging of RPE cells using two-photon excitation [7569-74]

L. Zhao, D. Chen, J. Qi, J. Qu, Shenzhen Univ. (China)

7569 2C In vivo multiphoton imaging of obstructive cholestasis in mice [7569-75]

F.-C. Li, Y. Y. Lee, L.-L. Chiou, H.-S. Lee, C.-Y. Dong, National Taiwan Univ. (Taiwan)

7569 2D Versatile photonic crystal fiber-enabled source for multi-modality biophotonic imaging beyond conventional multiphoton microscopy [7569-77]

H. Tu, S. A. Boppart, Univ. of Illinois at Urbana-Champaign (United States)

$75692 \mathrm{~F} \quad$ Multiplex coherent anti-Stokes Raman scattering flow cytometry for real-time classification of particles in a microfluidic channel [7569-79]

C. H. Camp, Jr., S. Yegnanarayanan, A. A. Eftekhar, Georgia Institute of Technology (United States); H. Sridhar, Harvard Univ. (United States); A. Adibi, Georgia Institute of Technology (United States)

$75692 \mathrm{G}$ Quantitative analysis of biological tissues using Fourier transform-second-harmonic generation imaging [7569-80]

R. Ambekar Ramachandra Rao, M. R. Mehta, K. C. Toussaint, Jr., Univ. of Illinois Urbana-Champaign (United States)

$756921 \quad$ Fluorescence performance standards for confocal microscopy [7569-82]

S. Rüttinger, Physikalisch-Technische Bundesanstalt (Germany); P. Kapusta, V. Völlkopf,

F. Koberling, R. Erdmann, PicoQuant GmbH (Germany); R. Macdonald,

Physikalisch-Technische Bundesanstalt (Germany)

7569 2J Fiber-based multiphoton system [7569-83]

G. Liu, Beckman Laser Institute (United States) and Univ. of California, Irvine (United States); K. Kieu, F. W. Wise, Cornell Univ. (United States); Z. Chen, Beckman Laser Institute (United States) and Univ. of California, Irvine (United States) 
$75692 \mathrm{R} \quad$ In vivo deep tissue imaging with long wavelength multiphoton excitation (Best Poster Award) [7569-92]

D. Kobat, M. E. Durst, N. Nishimura, A. W. Wong, C. B. Schaffer, C. Xu, Cornell Univ. (United States)

$75692 S$ Coherent anti-Stokes Raman scattering microscopy using photonic crystal fibers [7569-93] M. Naji, S. Murugkar, K. R. Khan, H. Anis, Univ. of Ottawa (Canada)

$75692 \mathrm{~T} \quad$ High-resolution imaging of a corneal incision by second- and third-harmonic generation microscopy [7569-94]

L. Jay, Maisonneuve-Rosemont Hospital Research Ctr. (Canada), Univ. of Montreal (Canada), and Institut National de la Recherche Scientifique (Canada); C. Dion, Maisonneuve-Rosemont Hospital Research Ctr. (Canada) and Institut National de la Recherche Scientifique (Canada); A. Brocas, Lab. Laser, Plasmas et Procédés Photoniques (France); K. Singh, Maisonneuve-Rosemont Hospital Research Ctr. (Canada) and Institut National de la Recherche Scientifique (Canada); J.-C. Kieffer, Institut National de la Recherche Scientifique (Canada); I. Brunette, Maisonneuve-Rosemont Hospital Research Ctr. (Canada) and Univ. de Montréal (Canada); T. Ozaki, Maisonneuve-Rosemont Hospital Research Ctr. (Canada) and Institut National de la Recherche Scientifique (Canada)

7569 2V Broadband multiplex CARS microspectroscopy in the picosecond regime [7569-98] S. Michel, Institut Fresnel, CNRS, Aix-Marseille Univ. (France); A. Courjaud, Amplitude Systemes (France); J. Dudley, Institut FEMTO, CNRS (France); C. Finot, Institut Carnot de Bourgogne, CNRS, Univ. de Bourgogne (France); E. Mottay, Amplitude Systèmes (France); H. Rigneault, Institut Fresnel, CNRS, Aix-Marseille Univ. (France)

7569 2W Polarization second harmonic generation (PSHG) imaging of neurons: estimating the effective orientation of the SHG source in axons [7569-99]

S. Psilodimitrakopoulos, Institut de Ciències Fotòniques (Spain); V. Petegnief, G. Soria, Institut de Recerca Biomèdica de Barcelona (Spain); I. Amat-Roldan, Institut de Ciències Fotòniques (Spain); D. Artigas, Institut de Ciències Fotòniques (Spain) and Univ. Politècníca de Catalunya (Spain); A. M. Planas, Institut de Recerca Biomèdica de Barcelona (Spain); P. Loza-Alvarez, Institut de Ciències Fotòniques (Spain)

75692 Complementary equipment for controlling multiple laser beams on single scanner MPLSM systems [7569-101]

P. J. Helm, G. Nase, P. Heggelund, T. Reppen, Univ. of Oslo (Norway)

$75692 Z$ Investigating the protective properties of milk phospholipids against ultraviolet light exposure in a skin equivalent model [7569-102]

A. Russell, A. Laubscher, R. Jimenez-Flores, L. H. Laiho, California Polytechnic State Univ. (United States)

756930 In vivo tissue imaging using a compact mobile nonlinear microscope [7569-104] R. Cicchi, D. Kapsokalyvas, D. Stampouli, V. De Giorgi, D. Massi, T. Lotti, F. S. Pavone, Univ. degli Studi di Firenze (Italy) 
756931 Fast rasterscanning enables FLIM in macroscopic samples up to several centimeters [7569-106]

F. Koberling, V. Buschmann, PicoQuant GmbH (Germany); C. Hille, Univ. of Potsdam (Germany); M. Patting, PicoQuant GmbH (Germany); C. Dosche, Univ. of Potsdam (Germany); A. Sandberg, A. Wheelock, Karolinska Institutet (Sweden); R. Erdmann, PicoQuant GmbH (Germany)

756932 Extracellular oxygen concentration mapping with a confocal multiphoton laser scanning microscope and TCSPC card [7569-108]

N. A. Hosny, D. A. Lee, M. M. Knight, Queen Mary Univ. of London (United Kingdom)

756933 A multimodal multiphoton microscope for biological imaging [7569-109]

R. Mouras, A. Downes, G. Rischitor, M. Mari, A. Elfick, Univ. of Edinburgh (United Kingdom)

756937 Non-linear 3D microscopy of ex-vivo corneas [7569-113]

J. M. Bueno, E. J. Gualda, P. Artal, Univ. de Murcia (Spain)

756939 Annular aperture detection scheme in radially polarized coherent anti-Stokes Raman scattering (RP-CARS) microscopy for contrast improvement [7569-126]

J. Lin, F. LU, National Univ. of Singapore (Singapore); H. Wang, Data Storage Institute, A*STAR

(Singapore); W. Zheng, C. J. R. Sheppard, Z. Huang, National Univ. of Singapore (Singapore)

7569 3A Scheme for efficient fiber-based CARS probe [7569-130]

M. Balu, G. Liu, Beckman Laser Institute and Medical Clinic (United States); Z. Chen,

B. J. Tromberg, E. O. Potma, Beckman Laser Institute and Medical Clinic (United States) and Univ. of California, Irvine (United States)

Author Index 
Downloaded From: https://www.spiedigitallibrary.org/conference-proceedings-of-spie on 26 Apr 2023

Terms of Use: https://www.spiedigitallibrary.org/terms-of-use 


\title{
Conference Committee
}

\author{
Symposium Chairs
}

James G. Fujimoto, Massachusetts Institute of Technology (United States)

R. Rox Anderson, Wellman Center for Photomedicine, Massachusetts General Hospital (United States), and Harvard School of Medicine (United States)

Program Track Chairs

Ammasi Periasamy, University of Virginia (United States)

Daniel L. Farkas, Cedars-Sinai Medical Center (United States)

Conference Chairs

Ammasi Periasamy, University of Virginia (United States)

Peter T. C. So, Massachusetts Institute of Technology (United States)

Karsten König, Saarland Universität (Germany)

\section{Program Committee}

Wolfgang Becker, Becker \& Hickl GmbH (Germany)

Keith M. Berland, Emory University (United States)

Guy C. Cox, The University of Sydney (Australia)

Alberto Diaspro, Università degli Studi di Genova (Italy)

Chen-Yuan Dong, National Taiwan University (Taiwan)

Dennis Donley, Olympus America (United States)

Kevin W. Eliceiri, University of Wisconsin, Madison (United States)

Scott E. Fraser, California Institute of Technology (United States)

Paul M. French, Imperial College London (United Kingdom)

Hans C. Gerritsen, Utrecht Universiteit (Netherlands)

Min Gu, Swinburne University of Technology (Australia)

Stefan W. Hell, Max-Planck-Institut für biophysikalische Chemie (Germany)

Brian A. Herman, The University of Texas Health Science Center at San Antonio (United States)

Satoshi Kawata, Osaka University (Japan)

Arnd K. Krueger, Newport Spectra-Physics (United States)

Joseph R. Lakowicz, University of Maryland School of Medicine (United States)

Stephen M. McDonald, Coherent, Inc. (United States)

Simon C. Watkins, University of Pittsburgh (United States)

Paul W. Wiseman, McGill University (Canada) 
Sunney X. Xie, Harvard University (United States)

Bernhard Zimmermann, Carl Zeiss Jena GmbH (Germany)

Warren R. Zipfel, Cornell University (United States)

Session Chairs

Keynote Session

Ammasi Periasamy, University of Virginia (United States)

1 Raman/CARS Microscopy I

Sunney X. Xie, Harvard University (United States)

2 Raman/CARS Microscopy II

Ji-Xin Cheng, Purdue University (United States)

3 Raman/CARS Microscopy III

Hervé Rigneault, Institut Fresnel (France)

4 FLIM/FRET/FCS I

Angelika C. Rueck, Universität Ulm (Germany)

5 FLIM/FRET/FCS II

Hans C. Gerritsen, Utrecht Universiteit (Netherlands)

6 Technology Development and Applications I

Peter T. C. So, Massachusetts Institute of Technology (United States)

7 Second Harmonic Generation I

Chen-Yuan Dong, National Taiwan University (Taiwan)

8 Second Harmonic Generation II

Paul J. Campagnola, University of Connecticut Health Center (United States)

9 Technology Development and Applications II

Karsten König, JenLab GmbH (Germany)

Poster Session

Vladimir Ghukasyan, National Yang-Ming University (Taiwan)

Eric O. Potma, University of California, Irvine (United States)

Kevin W. Eliceiri, University of Wisconsin-Madison (United States) 